\title{
The mitochondrial biogenesis signaling pathway is a potential therapeutic target for myasthenia gravis via energy metabolism (Review)
}

\author{
LINGLING KE ${ }^{1,2}$, QING LI $^{1,2}$, JINGWEI SONG ${ }^{1,2}$, WEI JIAO $^{1,2}$,

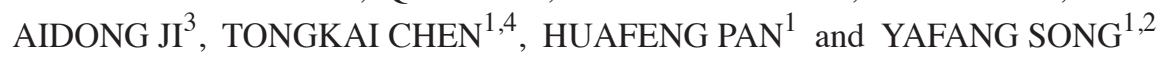 \\ ${ }^{1}$ Science and Technology Innovation Center, ${ }^{2}$ Institute of Pi-Wei, Guangzhou University of Chinese Medicine, \\ Guangzhou, Guangdong 510405; ${ }^{3}$ Clinical Medical College of Acupuncture, Moxibustion and Rehabilitation, \\ Guangzhou University of Chinese Medicine, Guangzhou, Guangdong 510006; ${ }^{4}$ Institute of Clinical Pharmacology, \\ Guangzhou University of Chinese Medicine, Guangzhou, Guangdong 510405, P.R. China
}

Received February 18, 2020; Accepted July 21, 2020

DOI: $10.3892 / \mathrm{etm} .2021 .10134$

\begin{abstract}
Myasthenia gravis (MG) is an autoantibody-mediated autoimmune disease that is characterized by muscle weakness and fatigue. Traditional treatments for MG target the neuromuscular junction (NMJ) or the immune system. However, the efficacy of such treatments is limited, and novel therapeutic options for MG are urgently required. In the current review, a new therapeutic strategy is proposed based on the mitochondrial biogenesis and energy metabolism pathway, as stimulating mitochondrial biogenesis and the energy metabolism might alleviate myasthenia gravis. A number of cellular sensors of the energy metabolism were investigated, including AMP-activated protein kinase (AMPK) and sirtuin 1 (SIRT1). AMPK and SIRT1 are sensors that regulate cellular energy homeostasis and maintain energy metabolism by balancing anabolism and catabolism. Peroxisome proliferator-activated receptor $\gamma$ coactivator $1 \alpha$ and its downstream transcription factors nuclear respiratory factors 1 , nuclear respiratory factors 2 , and transcription factor A are key sensors of mitochondrial biogenesis, which can restore mitochondrial DNA and produce new mitochondria. These processes help to control muscle contraction and relieve the symptoms of MG, including muscle weakness caused by dysfunctional NMJ transmission. Therefore, the present review
\end{abstract}

Correspondence to: Professor Yafang Song, Institute of Pi-Wei, Guangzhou University of Chinese Medicine, 12 Airport Road, Guangzhou, Guangdong 510405, P.R. China

E-mail: Stephanie237@163.com

Professor Huafeng Pan, Science and Technology Innovation Center, Guangzhou University of Chinese Medicine, 12 Airport Road, Guangzhou, Guangdong 510405, P.R. China

E-mail: gzphf@gzucm.edu.cn

Key words: myasthenia gravis, mitochondria, energy metabolism, mitochondrial biogenesis, AMP-activated protein kinase, proliferator-activated receptor $\gamma$ coactivator $1 \alpha$ provides evidence for the therapeutic potential of targeting mitochondrial biogenesis for the treatment of MG.

\section{Contents}

1. Introduction

2. Mitochondrial dysfunction disorders

3. Regulatory mechanism of mitochondrial biogenesis on MG through energy metabolism

4. Effect of mitochondrial biogenesis on MG is based on energy metabolism

5. Conclusions

\section{Introduction}

Myasthenia gravis (MG) is an autoimmune disease that is mediated by autoantibodies targeting acetylcholine receptors (AChRs) at the neuromuscular junction (NMJ), ultimately causing damage to skeletal muscles (1). The clinical symptoms of MG include blepharoptosis, muscle weakness, slurred speech and dysphagia, and may also include paralysis of respiratory muscles in severe cases (2-4). Globally, the prevalence of MG is $15-30$ per 100,000 , with an annual incidence of $>1$ per 100,000 (5). The mean duration of the myasthenia crisis course is 42 days and, if left untreated, fatality rates may reach $80 \%(6,7)$. However, with the advances in medical knowledge, MG-associated worldwide mortality has been reduced from $70 \%$ in the 1930 s to $30 \%$ by 1955 , and is currently $<10 \%$ (8).

To date, treatments for MG are limited to the NMJ or the immune system, and acetylcholinesterase inhibitors are the first-line treatment of MG (9). Neostigmine and pyridostigmine are the most commonly used anticholinesterase drugs in the clinical setting (10). Acetylcholinesterase inhibitors compensate for the degradation of acetylcholine (Ach) and pernicious effects on the NMJ by increasing the concentration of $\mathrm{ACh}$ in the synaptic cleft (11). However, this class of drugs can only alleviate symptoms in a minority of the patients, and cannot 
further modify the autoimmune response (12). Therefore, the majority of the patients require adjuvant and synergistic therapy. At present, glucocorticoids are the most effective immunosuppressant adjuvant for the treatment of MG (5). However, due to serious adverse reactions caused by high-dose shock therapy over a short period of time, transient myasthenia aggravation may occur (13). Occasionally, chemical immunosuppressants, including azathioprine, are used in combination therapy, which not only enhance the curative effect, but also help to reduce hormone dosage (14). Although most patients exhibit good tolerance to azathioprine, the effect of the drug is slow and, due to the immunosuppression, patients may be at an increased risk of liver damage and bone marrow suppression, amongst other adverse effects (15). Moreover, it has been demonstrated that the levels of choline acetyltransferase in the thymus of patients with MG and thymic hyperplasia were higher compared with those in control subjects (16), which suggested that an abnormal immune tolerance in the thymus serves an important role in the pathogenesis of MG. Therefore, thymectomy or thymic radiotherapy is a common therapy used for patients with $\mathrm{MG}$, and a previous study reported that $\sim 30 \%$ of patients achieved complete and stable remission or pharmacological remission following this surgery (12). However, thymectomy may also disrupt self-tolerance and further lead to immune system dysfunction. Immunomodulatory treatments are promising for the reduction or elimination of $\mathrm{MG}$ symptoms (14). Plasma exchange can alleviate symptoms by removing the pathogenic substances in the plasma and reducing the concentration of autoantibodies (17). Effects may be observed from 2 to 5 days following treatment $(18,19)$, but plasma exchange only alleviates MG temporarily by removing harmful substances from the blood. Additionally, plasma exchange may be associated with various complications, such as plasma anaphylaxis (20). Intravenous immune globulin (IVIG) is often used in the emergency treatment of severe myasthenia, and has the advantages of being rapid, effective and relatively low-cost (21). However, IVIG is associated with potential risks of infection and thrombosis (22) (Table I).

Although there are a number of treatment options for $\mathrm{MG}$, $\sim 10 \%$ of patients are treatment-intolerant, and up to $80 \%$ of patients do not achieve complete stabilization (23). The onset of MG is associated with AChR antibody (AChR-Ab), but there is no clear association between disease severity and antibody titer (24). Moreover, it has been demonstrated that both neuronal and muscular cells in MG are highly sensitive to energy deprivation, which significantly affects signal transmission and the normal physiological function of the NMJ. Therefore, by investigating mitochondrial biogenesis and energy metabolism pathways, the current review puts forward the hypothesis that modulation of mitochondrial biogenesis may represent a viable therapeutic option MG by regulating energy metabolism, as well as the dysfunctional NMJ transmission.

\section{Mitochondrial dysfunction disorders}

Previous studies investigating neuromuscular diseases mainly focused on immune responses and synapses of the NMJ $(25,26)$. MG is considered to be a neuromuscular disease, and dysfunctional transmission at the NMJ and autoantibody binding appear to be the initial mechanisms of MG (27). The
NMJ is composed of Schwann cells, which are a highly specialized skeletal muscle cell membrane, and the axon terminal of the motor nerve (28). Damaging any part of this system may cause signal transmission disorders $(29,30)$. The NMJ can be targeted by a variety of autoimmune antibodies, including AChR-Ab, ryanodine receptor antibody, muscle-specific receptor tyrosine kinase antibody (MuSK-Ab) and low-density lipoprotein receptor-related protein 4 antibody (31). These antibodies bind to the postsynaptic membrane, and attack and destroy postsynaptic molecules. This damage to postsynaptic structures occurs by activation of the complement system, enhancing the binding capacity of the AChR or inhibition of the function of ACh, and subsequently induces clinical MG symptoms by reducing AChR numbers and disrupting clustering at muscle tubules (5).

Although the immune mechanisms underlying neuromuscular diseases, such as MG, has been extensively investigated, there are relatively few studies on muscle cell damage and mitochondrial dysfunction. MG autoantibodies do not only induce AChR destruction directly, but also cause intracellular mitochondrial changes (32). In a previous experimental autoimmune MG (EAMG) rat study, it was observed that both mitochondrial structure and skeletal muscle function were compromised to varying degrees (15). Mitochondria are the core source of cellular energy and are essential for the daily activities of hypermetabolic tissues, such as muscle contraction and utilization (33). Mitochondrial dysfunction may affect the normal energy metabolism and, in combination with specific autoantibodies, aggravate neuromuscular disorders (34). Kordas et al (24) demonstrated that the mitochondrial protein $\mathrm{CHCHD} 10$ is required for ATP production, which facilitates AChR expression and promotes agrin-induced AChR clustering.

Mitochondrial dysfunction and sensitivity of muscle cells to energy deprivation are major characteristics of neuromusculardiseases (35). It has been demonstrated that mitochondrial dysfunction serves an important role in muscular dystrophy muscle consumption (36). Excessive reactive oxygen species (ROS) produced by mitochondrial dysfunction may trigger autophagy, which results in muscle atrophy from an enhanced catabolism and decreased protein synthesis in the skeletal muscle (37). Blocking the extracellular ATP/P2X axis results in enhancement of muscle $\mathrm{T}$ regulatory cells and accelerates the muscular dystrophic process in mdx mice (38). Similarly, mitochondrial dysfunction has also been indicated to be one of the important pathogenetic mechanisms involved in amyotrophic lateral sclerosis (ALS) (39). Abnormal mitochondrial morphology and dysfunction have been previously identified in patients with ALS (40). In addition, it has been reported that resting energy consumption in patients with ALS was lower compared with that in healthy individuals using indirect calorimetry (41). Recently, a number of studies have demonstrated that MG is closely associated with dysfunction of mitochondria and muscle cells $(42,43)$. The muscle tissue of patients with MG display abnormal mitochondrial morphology and function, and the energy levels are often significantly lower than normal $(44,45)$. Similarly, abnormally shaped and structured mitochondria on muscle biopsy, ragged-red fibers, loss of mitochondrial respiratory chain complexes-1 and muscle aerobic dysfunction were observed in MG $(46,47)$. These previous findings suggest the 
Table I. Most frequently used treatments for myasthenia gravis.

\begin{tabular}{lll}
\hline Therapy & \multicolumn{1}{c}{ Drugs or methods } & Typical side effects \\
\hline Symptomatic therapy & $\begin{array}{l}\text { Acetylcholinesterase inhibitors } \\
\text { (Neostigmine, pyridostigmine) }\end{array}$ & Gastrointestinal illnesses \\
Immunosuppressive therapy & $\begin{array}{l}\text { Immunosuppressive drugs } \\
\text { (glucocorticoid, azathioprine) }\end{array}$ & Transient myasthenia \\
Thymectomy therapy & Thymectomy & Immune impairment \\
Supportive therapy & Physical activity and systematic training & Overwork aggravates illness \\
Immunomodulating therapy & Intravenous immune globulin & Allergic reactions \\
Plasma exchange & Plasma exchange & Plasma allergy
\end{tabular}

Table II. A selected list of neuromuscular diseases that exhibit mitochondrial dysfunction.

\begin{tabular}{|c|c|c|}
\hline Diseases & Mitochondrial dysfunction & (Refs.) \\
\hline MD & $\begin{array}{l}\text { Increased ROS and oxidative stress, increased catabolism of muscle } \\
\text { and protein degradation, triggering autophagy and decreased ATP }\end{array}$ & $(24-26)$ \\
\hline ALS & $\begin{array}{l}\text { Increased nutrition and fat mass, decreased respiratory parameters, } \\
\text { motor neuron involvement, and decreased metabolism }\end{array}$ & $(27,28)$ \\
\hline MG & $\begin{array}{l}\text { Decreased metabolism and energy level, mitochondrial respiratory } \\
\text { chain complex-1 loss, muscular atrophy, and increased inflammatory } \\
\text { responses }\end{array}$ & $(15,29-31)$ \\
\hline
\end{tabular}

MD, muscular dystrophy; ALS, amyotrophic lateral sclerosis; MG, Myasthenia gravis; ROS, reactive oxygen species.

presence of mitochondrial abnormalities in neuromuscular diseases, such as MG, which may lead to muscular weakness. Therefore, it is of great significance to further explore the mitochondrial biogenesis signaling pathway and its link to the energy metabolism as a potential target for the treatment of MG (Table II).

\section{Regulatory mechanism of mitochondrial biogenesis on MG through energy metabolism}

As aforementioned, mitochondrial dysfunction can damage the normal function of muscle, leading to the development of MG. However, the specific role of mitochondria in the pathogenesis of MG remains unclear. Herein, the current review discusses the potential mechanisms regulating mitochondrial function in MG from the perspective of biogenesis and energy metabolism.

The term 'energy metabolism' refers to a series of continuous, cyclic processes in which the energy substance ATP is produced, transported and utilized by the action of ATP synthase or ATP hydrolase, respectively (48). AMP-activated protein kinase (AMPK) is one of the key enzymes of the mitochondrial energy metabolism (49). AMPK can phosphorylate a variety of transcription factors included in the energy metabolism and biogenesis pathways, providing necessary cellular mechanisms for skeletal muscle plasticity (50).

AMPK: A key mitochondrial energy metabolism molecule. AMPK is a serine/threonine protein kinase that regulates cellular energy metabolism and serves a key role in maintaining the cellular energy balance (51). AMPK is an allotrimer that is composed of a catalytic $\alpha$ and regulatory $\beta$ and $\gamma$ subunits, which are assembled into a total of 12 possible AMPK complexes (52). In general, AMPK is activated by upstream kinases, including liver kinase $\mathrm{B} 1, \mathrm{Ca}^{2+} /$ calmodulin-dependent protein kinase 2 and transforming growth factor- $\beta$-activated kinase 1 (53). An increase in the cellular AMP:ATP and ADP:ATP ratio during low energy state activates AMPK by inhibiting the synthesis process that consumes ATP to restore energy balance, while promoting the decomposition process that produces ATP (Fig. 1) (15). The activation of AMPK triggers a cellular metabolic transformation, promoting an uptake of lipids and glucose to balance the energy metabolism of skeletal muscle (54). The mechanism is that AMPK inhibits glycogen synthesis by inhibiting the translation of TBC1 domain family member 1 and glucose transporter type 4 (GLUT4), and fatty acid (FA) synthesis by inhibiting acetyl-coenzyme A carboxylase and sterol regulatory element-binding protein-1c (51). 5-Aminoimidazole-4-carboxamide 1 - $\beta$-D-ribofuranoside (AICAR) acts as an AMP analogue and has been widely used as an AMPK activator and stimulant for mitochondrial biogenesis (55). Activating the AMPK signaling pathway through muscle training or using pharmacological AICAR treatment may increase the mitochondrial content in cells and tissues (56). Metformin exerts anti-inflammatory effects via AMPK. In an EAMG rat model, it was demonstrated that the oral administration of metformin attenuated MG severity by correcting the imbalance of different T-cell groups (57). 


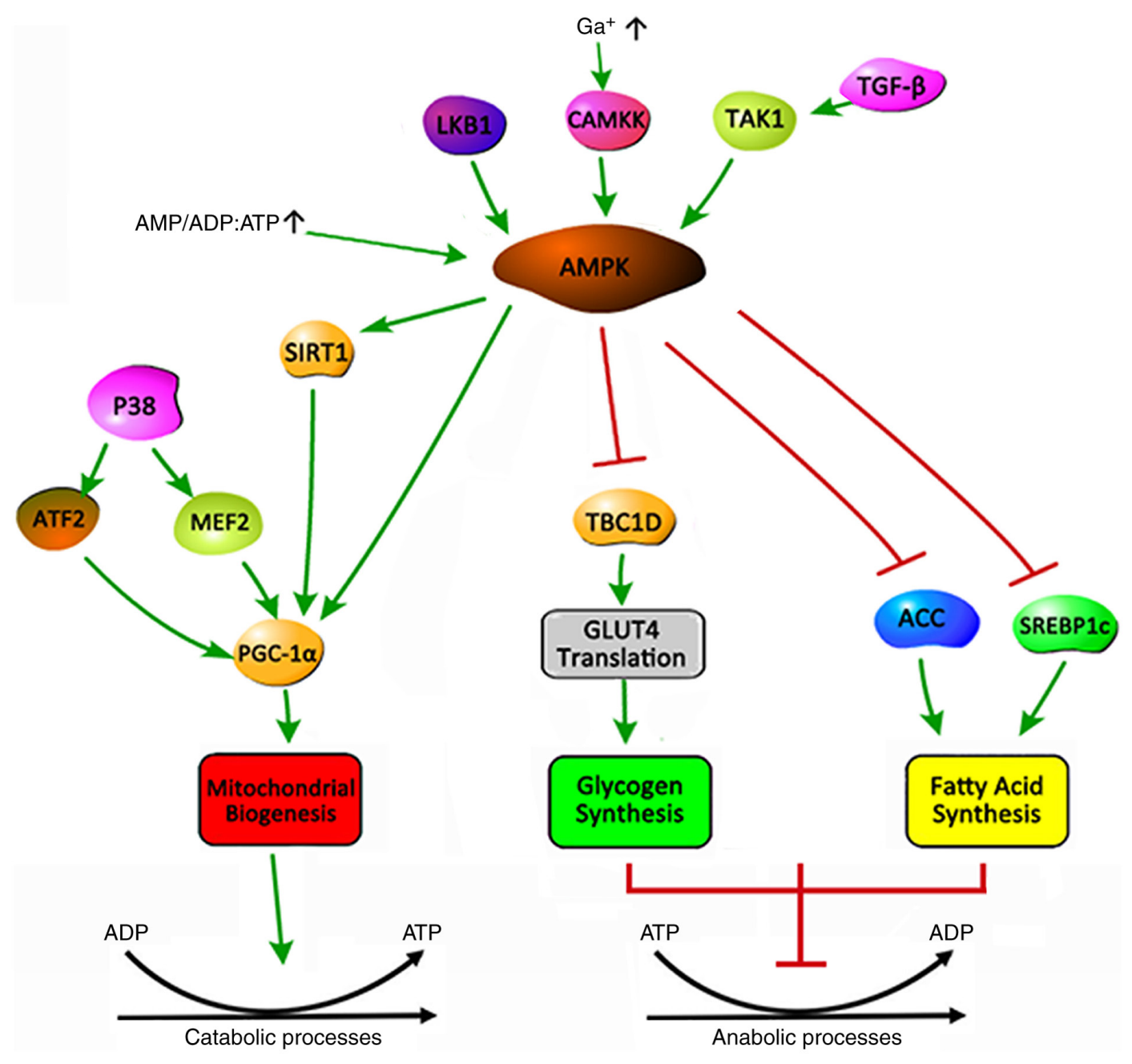

Figure 1. Balance of the energy metabolism triggered by activation of AMPK. AMPK can be activated by the upstream kinases LKB1, CAMKK2, TAK1 and high AMP/ADP:ATP ratios, which maintain the body's energy balance by promoting catabolism and inhibiting anabolism. (1) Promoting catabolism: AMPK controls the biogenesis through phosphorylation of SIRT1 to activate PGC-1 $\alpha$, thereby affecting the energy metabolism of the body. P38 can also activate PGC-1 $\alpha$ by phosphorylating ATF2 and MEF2. (2) Inhibition of anabolism: AMPK inhibits glycogen synthesis by inhibiting TBC1D1 to control the translation of GLUT4. AMPK inhibits fatty acid synthesis by suppressing ACC and SREBP1C. Arrow, activation. Bar-headed line, inhibition. AMPK, AMP-activated protein kinase; LKB1, Liver Kinase B1; CAMKK2, calcium/calmodulin dependent protein kinase kinase 2; TAK1, TGF-beta-activated kinase 1; SIRT1, sirtuin 1; PGC-1 $\alpha$, proliferator-activated receptor $\gamma$ coactivator 1 $\alpha$; ATF2, activating transcription factor 2; MEF2, myocyte enhancer factor 2; GLUT4, glucose transporter type 4; TBC1D1, Tre-2/BUB2/cdc1 domain family 1; ACC, acetyl-CoA carboxylase; SREBP1C, sterol-regulatory element binding protein $1 \mathrm{C}$.

Sirtuin 1 (SIRT1): A mitochondrial energy metabolism sensor. SIRT1 is a member of the NAD ${ }^{+}$-dependent histone deacetylase family and is widely considered to be a cellular energy sensor (58). SIRT1 has received widespread attention as it is positively regulated by the oxidized coenzyme $\mathrm{NAD}^{+}$, and due to its ability to regulate peroxisome proliferator-activated receptor $\gamma$ coactivator $1 \alpha(\mathrm{PGC}-1 \alpha)$ and other mitochondrial-related genes (59). SIRT1 activation is triggered by an increased $\mathrm{NAD}^{+} / \mathrm{NADH}$ ratio (60). The overexpression of brain-specific SIRT1 in aged mice results in more youthful NMJ morphological characteristics (distinct terminal Schwann cells, clear innervation, and pretzel-shaped motor endplates), with increased AChR numbers and NMJ innervation (61). It has also been reported that SIRT1 exerts protective effects during metabolic syndrome (62). Deletion of SIRT1 reduces mitochondrial biogenesis and ATP production, while SIRT1 overexpression increases the expression of PGC-1, which is a regulatory factor of mitochondrial biogenesis (63).
Association between AMPK and SIRT1. The process of mitochondrial biogenesis is strictly regulated by a series of upstream energy metabolism pathways, including AMPK and SIRT1, and the regulation of PGC- $1 \alpha$, nuclear respiratory factors (Nrfs) and transcription factor A (TFAM) via downstream effectors (Fig. 2) (64). AMPK promotes mitochondrial biogenesis in three ways: i) It increases the activity or expression of the transcriptional coactivator PGC-1 $\alpha$; ii) it enhances the deacetylation of AMPK through SIRT; or iii) it directly phosphorylates itself, which has a positive effect on its expression levels (65). Recently, the activation of AMPK has been demonstrated to activate and facilitate the deacetylation of PGC-1 by phosphorylating Thr177/Ser538 of PGC-1 $\alpha$ (48). P38-MAPK can directly activate the upstream transcription factors of the PGC-1 $\alpha$ gene, such as activating transcription factor 2 and myocyte enhancer-binding factor 2 , and promote the transcription and activity of the PGC- $1 \alpha$ protein (66). In addition to PGC- $1 \alpha$ and SIRT1, AMPK can phosphory- 


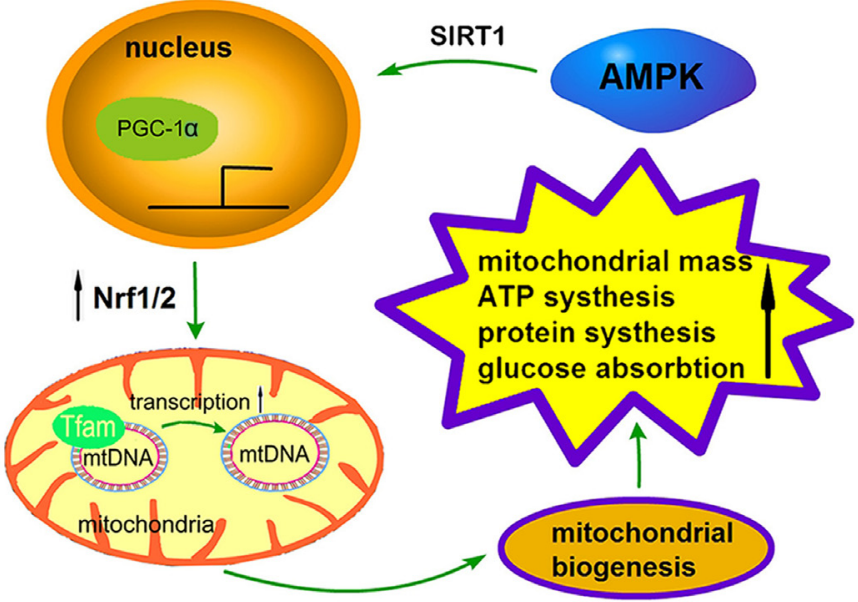

Figure 2. A sensor network regulating mitochondrial biogenesis. AMPK activation promotes the expression of PGC- $1 \alpha$ and its downstream signals and serves a key role in mitochondrial biogenesis. The activation of AMPK by phosphorylation of SIRT1 enhances PGC-1a dependent transcription. PGC-1 $\alpha$ interacts with nuclear receptors and stimulates $\mathrm{Nrf} 1 / 2$ to participate in the expression of various nuclear coding genes and TFAM, leading to an increase of mtDNA replication transcribed on TFAM. These processes ultimately affect mitochondrial biogenesis, promoting mitochondrial mass, ATP synthesis, protein synthesis and glucose absorption. AMPK, AMP-activated protein kinase; SIRT1, sirtuin 1; PGC-1 $\alpha$, proliferator-activated receptor $\gamma$ coactivator $1 \alpha$; TFAM, transcription factor A; mtDNA, mitochondrial DNA.

late a number of different transcription factors, including Nrf1/2 and TFAM (67). The synergistic expression of these transcriptional regulators under AMPK stimulation provides the necessary support for the metabolism and biogenesis of skeletal muscle.

PGC-1 $\alpha$ : A major mitochondrial biogenesis regulator. PGC-1 $\alpha$ is a major regulator of diverse metabolic pathways and mitochondrial biogenesis, and serves an important role in mitochondrial biogenesis and gene expression (68). As a co-activator with low transcriptional activation activity, the activity of PGC-1 $\alpha$ is significantly enhanced by its interaction with nuclear receptors, which can regulate the expression of target genes (69). PGC-1 $\alpha$ controls several aspects of the muscular metabolism during exercise, and it is generally hypothesized that $\mathrm{PGC}-1 \alpha$ regulates respiratory function, controls muscle aerobic metabolism and mitochondrial biogenesis, and responds to environmental and physiological changes (70,71). PGC-1 $\alpha$ has been identified to serve a key role in regulating mitochondrial function, and is specialized for the thermogenesis and differentiation of cell type in brown adipose tissue $(72,73)$. Forced overexpression of PGC-1 $\alpha$ in cardiac myocytes in culture has been indicated to induce the expression of nuclear and mitochondrial genes that are involved in multiple mitochondrial energy transduction/production pathways, increase the number of cellular mitochondria and stimulate coupled respiration (74). In addition, PGC- $1 \alpha$ is the detoxified cation of ROS that is generated during the mitochondrial respiration process (75). PGC-1 $\alpha$ knockout mice display marked hyperactivity accompanied by neuronal degeneration (76). Similarly, the expression of PGC-1 $\alpha$ was also found to be decreased in spinal muscular atrophy mouse models (77). PGC-1 $\alpha$ is expressed at high levels in tissues with an abundance of mitochondria and an active oxidative metabolism, allowing for a response to increased energy needs. In muscle, PGC- $1 \alpha$ activates mitochondrial biogenesis, increases FA oxidation and GLUT4 expression, which, in turn, reduces fat accumulation and increases insulin sensitivity (75).

Nrfs: Regulation of downstream transcription factors in mitochondrial biogenesis. Nrfs, which are the downstream nuclear receptors of PGC-1 $\alpha$, contain the recognition sites of mitochondrial DNA (mtDNA) promoter and serve a key role in biogenesis (78). As a mtDNA transcription factor, Nrfs can alleviate $\mathrm{MG}$ by regulating the mitochondrial respiratory chain function and alleviating antioxidant damage (79). Nrf-1 and Nrf-2 belong to the $\mathrm{CNC}$ basic leucine zipper (bZIP) regulatory protein family, are the major transcription factors in the human genome and powerful stimulators of the expression of nuclear genes required for mitochondrial respiratory function (80-82). Nrf-1 was initially indicated to be associated with the regulation of genes, serving a role in a wide range of biological functions, including signal transduction, organelle biogenesis, protein synthesis and cell growth (83). Nrf-1 also coordinates synaptic activity and energy metabolism by regulating excitatory neurotransmission via genes that code for subunits of the $\mathrm{N}$-methyl-D-aspartate receptor (84). Nrf-1 directly regulates the expression of nuclear encoded genes related to respiratory chain expression, assembly and function, or indirectly regulates the cytochrome oxidase subunit genes that are encoded by mitochondria, and is associated with the generation of ROS and oxidative stress (85). Recently, Nrf-2 was demonstrated to serve an important role in cellular bioenergetics by controlling substrate availability for mitochondrial respiration (46). In addition, Nrf-2 regulates mitochondrial biogenesis through co-activation with PGC-1 $\alpha$ and the expression of the TFAM gene (86). Nrf-1 and Nrf-2 are involved in the regulation of inflammatory processes and antioxidant pathways, acting protectively against ROS-induced toxicity (87). Nrf-2 has been reported to control oxidative stress or exercise-induced mitochondrial biogenesis by promoting Nrf-1 transcription following its binding to the antioxidant response element of the Nrf-1 promoter (88).

TFAM: Promoting mitochondrial biogenesis by regulating $m t D N A$. TFAM is a high mobility family protein factor that is located in mitochondria but encoded by nuclear genes (89). TFAM can be activated by PGC- $1 \alpha$ and Nrfs to transcribe and regulate mtDNA (90). In a study investigating mtDNA in patients with MG, mtDNA amplification was performed in 20 muscle samples of patients with muscle-specific kinase MG, and multiple mtDNA deletions were identified in 13 patients (91).

The function of TFAM in the maintenance and transcription initiation of mtDNA can be summarized by the following three aspects: i) TFAM regulates mtDNA copy number. As a major regulator of the mtDNA transcriptional machinery, TFAM directly promotes transcription utilizing the mitochondrial RNA polymerase (92). In a previous study, both the mRNA and protein levels of TFAM were assessed during muscle differentiation, and it was observed that TFAM protein and mRNA increased two-fold in parallel, corresponding to 
a two- to three-fold increase of mitochondrial content (93). Therefore, increasing levels of TFAM may promote the transcription of mtDNA and the production of proteins; ii) TFAM maintains mtDNA structural stability. Although mtDNA is a short molecule, mistakes often occur during replication, transcription and translation as mitochondria lack a DNA proofreading and repair system (94). TFAM avoids these errors to a certain extent. It has been demonstrated that TFAM binds to damaged DNA and regulates TFAM/DNA affinity by interacting with proteins, leading to tighter compression of mtDNA and reduced accessibility to transcription, replication or repair factors $(83,95)$. TFAM can shape DNA and compensate for the unstable phenotype of mtDNA, which is the key to maintaining the structure and stability of mtDNA. iii) TFAM prevents mtDNA damage. The mutation rate of mtDNA is 10-20 times higher compared with that of nuclear DNA (96); this is due to its high superoxide environment, lack of protective histone-like proteins and poor reparative activity in response to damage. It has been revealed that TFAM binds and coats mtDNA to protect it from ROS and degradation, while increasing mitochondrial function (92). The ratio of mtDNA molecules to TFAM protein molecule in cells is $\sim 1: 1,000$ (97). Furthermore, TFAM acts as a packaging protein to bind mtDNA, forming mtDNA-protein complexes and compressing a number of mtDNAs into the nucleoid (86).

Mitochondrial biogenesis based on energy metabolism is a tightly controlled process (98). When mitochondrial biogenesis is disrupted, the number of mitochondria and energy metabolism will be reduced, and mitochondrial gene and protein expression will be inhibited (99). It has been demonstrated that deletion of AMPK- $\alpha 1$ and AMPK- $\alpha 2$ in mouse skeletal muscle can affect FA utilization (100). Overexpression of TFAM in astrocytes induces mtDNA protection against A $\beta 1-42$ peptide, ultimately protecting neurons (101), while tissue-specific or partial deletion of the TFAM protein leads to respiratory chain defects (102). A previous study of miR-133a-deficient mice have suggested that the transcription of the mitochondrial biogenesis regulators PGC-1 $\alpha$, Nrf-1 and TFAM was reduced in miR-133a-deficient muscle, which was consistent with lower mitochondrial mass and impaired exercise capacity (103). In summary, these studies indicated that mitochondrial biogenesis is an indispensable process for maintaining mitochondrial function and normal muscular activity by regulating the stability of involved proteins and mtDNA.

\section{Effect of mitochondrial biogenesis on MG is based on energy metabolism}

It is known that mitochondrial biogenesis, which is regulated by energy metabolism, is the foundation for maintaining mitochondrial function and a normal muscular metabolism (104). When failure of mitochondrial biogenesis and energy metabolism occurs, individuals are prone to tissue dysfunction and degeneration $(105,106)$. Mitochondrial biogenesis or energy metabolism disorders have been observed in a number of diseases [such as epilepsy and Parkinson's disease (PD) involving nerve or muscle dysfunction $(107,108)$.

Effect of mitochondrial energy metabolism on MG. The adaptation of mitochondria to exercise is generally referred to as mitochondrial energy metabolism (109). The physiological function of mitochondria is to improve metabolic efficiency through oxidative phosphorylation of ATP, in order to supply energy for the contraction of muscle cells (104). Muscle contraction utilizes energy supplied by cells due to ATP decomposition to overcome resistance, converting chemical energy to mechanical energy (110). However, the mitochondrial energy metabolism is inevitably accompanied by ROS generation. Excessive ROS accumulation can cause irreversible oxidative stress damage to mitochondria and muscles (111).

In recent years, there has been accumulating evidence that the mitochondrial energy metabolism is crucial for the maintenance and plasticity of motor neurons, the NMJ and skeletal muscles(112) A variety of neurodegenerative diseases, including Alzheimer's disease (AD), PD and Huntington's disease, are associated with increased ROS production, mitochondrial dysfunction and apoptosis activation (113). Mitochondrial dysfunction and elevated ROS levels are common results in a number of neurodegenerative diseases and axonopathies, such as multiple sclerosis (114) and Charcot-Marie-Tooth disease (115). It is well known that excessive ROS induces inflammatory cytokine production and T-cell activation (116). In patients with MG, the levels of pro-inflammatory cytokines, including interleukin (IL)-6, IL-17 and interferon- $\gamma$, which are secreted by $\mathrm{T}$ effector cells, are increased (117). During the early stages of MG, exercise intolerance, muscular and nervous system dysfunction are commonly observed, amongst other clinical symptoms, which are similar to the clinical observations of mitochondrial myopathy, respiratory chain and energy metabolism disorders (118). A disruption of integrity of the energy metabolism at the NMJ is associated with the occurrence and development of MG (119). Neurite outgrowth is an energy consuming process: The energy metabolism provides enough energy to support protein and membrane synthesis at the NMJ, as well as intracellular transport (120). Aging NMJs exhibit abnormal morphology and decreased numbers of mitochondria, oxidative damage and reduced ATP synthesis (28).

Effect of mitochondrial biogenesis on MG. Biogenesis is the general term used to indicate an assimilation reaction in organisms, and it is a vital regulatory process of protein synthesis (121). As a semi-autonomous organelle, mitochondria serve an indispensable role in protein biogenesis (122). Proteins are the major effectors of cellular activities, and promote metabolism, maintain the composition of all cells, including muscle and nerves, and regulate the physiological functions of the body (123).

Misfolded, conformationally modified proteins can cause neurodegenerative diseases, such as AD and PD (considered as protein conformation disorders) (124). It has been demonstrated that insufficient protein levels make it difficult to maintain normal motor neuron function and survival (125). Loss of axonal domain proteins in nerve conduction may have a severe impact on neuromuscular integrity and health. For example, compromised axonal structural integrity may lead to pathological changes of peripheral nervous system myelinated fibers and muscle pathology (126). It has been demonstrated that impaired mitochondrial function of presynaptic and postsynaptic cells at the NMJ may cause neuromuscular dysfunction, 
mainly by reducing the production of presynaptic ACh and the number of postsynaptic AChRs (127). Caveolin-3 is an integral membrane protein that is essential for the repair of muscle membrane damage and is expressed in skeletal, cardiac and smooth muscle cells. It has been demonstrated that partial loss of caveolin-3 expression is a relatively common occurrence in the muscles of patients with MG. Caveolin-3 overexpression induced after NMJ defects in MG muscles exerts a protective effect on skeletal myotubes (128). It has been indicated that complement-dependent lysis of the post-synaptic membrane, receptor internalization, as well as direct interference with the binding capacity of ACh to the AChR, may cause muscular damage in patients with MG (129). The normal function of the low-density lipoprotein receptor-related protein 4 and clustering of proteins serve a key role in the proper formation and maintenance of the NMJ (130).

These findings suggest that the abnormalities of mitochondrial biogenesis and the energy metabolism are closely associated with the pathogenesis of MG. Regulation of mitochondrial function and the restoration of the function of related targets in the mitochondrial biogenesis pathway may represent a novel therapeutic approach to $\mathrm{MG}$ and similar muscle contraction disorders.

\section{Conclusions}

Although recent studies have investigated MG, the majority of these studies have focused on immunity $(22,131)$, while relatively few studies to date assessed the link between MG and mitochondrial function. As the powerhouse of the cell, mitochondria are the main energy source for skeletal muscle cells, enabling various biological processes and providing muscle movement support, and are key organelles for the maintenance of signal transmission and normal physiological function. Mitochondrial dysfunction directly affects the energy metabolism of skeletal muscle, impairing ACh biogenesis and NMJ signal transmission, ultimately accelerating the progression of MG. Therefore, it may be inferred that the mitochondrial function pathway may represent a promising target for the development of neuromuscular drugs for the treatment of MG.

Mitochondria and bioenergetic dysfunction are increasingly considered to be key components of neuromuscular diseases. In a number of neuromuscular diseases, it has been demonstrated that nerves and muscles are highly sensitive to energy deprivation, which has an adverse impact on recovery $(35,132)$. The mitochondrial biogenesis pathway involves multiple targets, including AMPK, SIRT1, PGC-1a, NRFs and TFAM, each of which serves an important regulatory role in neuromuscular function (133). Dysfunction of these factors may be involved in the development of neuromuscular diseases, such as MG, which are sensitive to energy deprivation and motor dysfunction.

Although the mitochondrial biogenesis pathway exhibits potential for the treatment of neuromuscular diseases, no specific pharmacological activator has yet been developed for the treatment of related diseases. A large number of researchers are currently investigating this approach in order to identify more effective treatment strategies. In summary, the biogenesis pathway represents a reasonable and feasible potential therapeutic target for neuromuscular diseases.

\section{Acknowledgements}

Not applicable.

\section{Funding}

The current study was supported by the National Science Foundation of China (grant no. 81473568) and Science Program for Overseas Scholar of Guangzhou University of Chinese Medicine (grant no. XH20160106).

\section{Availability of data and materials}

Not applicable.

\section{Authors' contributions}

LK, HP and YS designed the present study. LK, QL and TC prepared the draft of the manuscript; JS and WJ reviewed and edited the manuscript; AJ produced the figures; QL, HP and YS are responsible for text layout. All authors read and approved the final version of the manuscript.

\section{Ethics approval and consent to participate}

Not applicable.

\section{Patient consent for publication}

Not applicable.

\section{Conflicting interests}

The authors declare that they have no competing interests.

\section{References}

1. Cataneo AJM, Felisberto G Jr and Cataneo DC: Thymectomy in nonthymomatous myasthenia gravis-systematic review and meta-analysis. Orphanet J Rare Dis 13: 99, 2018.

2. Barnett C, Bril V, Kapral M, Kulkarni A and Davis AM Development and validation of the myasthenia gravis impairment index. Neurology 87: 879-886, 2016.

3. Gwathmey KG and Burns TM: Myasthenia gravis. Semin Neurol 35: 327-339, 2015.

4. Wang Z and Yan YP: Immunopathogenesis in myasthenia gravis and neuromyelitis optica. Front Immunol 8: 1785, 2017.

5. Gilhus NE, Skeie GO, Romi F, Lazaridis K, Zisimopoulou P and Tzartos S: Myasthenia gravis-autoantibody characteristics and their implications for therapy. Nat Rev Neurol 12: 259-268, 2016.

6. Juel VC: Myasthenia gravis: Management of myasthenic crisis and perioperative care. Semin Neurol 24: 75-81, 2004.

7. Thomas CE, Mayer SA, Gungor Y, Swarup R, Webster EA, Chang I, Brannagan TH, Fink ME and Rowland LP: Myasthenic crisis: Clinical features, mortality, complications, and risk factors for prolonged intubation. Neurology 48: 1253-1260, 1997.

8. Mantegazza $\mathrm{R}$ and Antozzi $\mathrm{C}$ : When myasthenia gravis is deemed refractory: Clinical signposts and treatment strategies. Ther Adv Neurol Disord 11: 1756285617749134, 2018.

9. Mehndiratta MM, Pandey S and Kuntzer T: Acetylcholinesterase inhibitor treatment for myasthenia gravis. Cochrane Database Syst Rev 16: CD006986, 2011.

10. Watanabe G, Yuki T, Sugaya R, et al: Effectiveness of treatment based on the simultaneous administration of pyridostigmine, prednisolone, calcineurin inhibitor, and intravenous immunoglobulin (PPCI therapy) in patients with myasthenia gravis. Eur J Neurol 25: 143-143, 2018. 
11. Luo $\mathrm{J}$ and Lindstrom J: AChR-specific immunosuppressive therapy of myasthenia gravis. Biochem Pharmacol 97: 609-619, 2015.

12. Binks S, Vincent A and Palace J: Myasthenia gravis: A clinical-immunological update. J Neurol 263: 826-834, 2016.

13. Wang L, Xi J, Zhang S, Wu H, Zhou L, Lu J, Zhang T and Zhao C: Effectiveness and safety of tacrolimus therapy for myasthenia gravis: A single arm meta-analysis. J Clin Neurosci 63: 160-167, 2019.

14. Gilhus NE and Verschuuren JJ: Myasthenia gravis: Subgroup classification and therapeutic strategies. Lancet Neurol 14: 1023-1036, 2015.

15. Song JW, Lei XW, Jiao W, Song Y, Chen W, Li J and Chen Z: Effect of Qiangji Jianli decoction on mitochondrial respiratory chain activity and expression of mitochondrial fusion and fission proteins in myasthenia gravis rats. Sci Rep 8: 8623, 2018.

16. Li YL, Li L and Li JM: Proteomic analysis of 11000 bands in thymic hyperplasia tissues of patients with myasthenia gravis. J Zhengzhou Univ 43: 291-295, 2012.

17. Guptill JT, Juel VC, Massey JM, Anderson AC, Chopra M, Yi JS Esfandiari E, Buchanan T, Smith B, Atherfold P, et al: Effect of therapeutic plasma exchange on immunoglobulins in myasthenia gravis. Autoimmunity 49: 472-479, 2016

18. Alipour-Faz A, Shojaei M, Peyvandi H, Ramzi D, Oroei M, Ghadiri F and Peyvandi M: A comparison between IVIG and plasma exchange as preparations before thymectomy in myasthenia gravis patients. Acta Neurol Belg 117: 245-249, 2017.

19. Newsom-Davis J, Wilson SG, Vincent A and Ward CD Long-term effects of repeated plasma exchange in myasthenia gravis. Lancet 1: 464-468, 1979.

20. Guptill JT, Oakley D, Kuchibhatla M, Guidon AC, Hobson-Webb LD, Massey JM, Sanders DB and Juel VC: A retrospective study of complications of therapeutic plasma exchange in myasthenia. Muscle Nerve 47: 170-176, 2013.

21. Furlan JC, Barth D, Barnett C and Bril V: Cost-minimization analysis comparing intravenous immunoglobulin with plasma exchange in the management of patients with myasthenia gravis Muscle Nerve 53: 872-876, 2016.

22. Mantegazza R, Bernasconi P and Cavalcante P: Myasthenia gravis: From autoantibodies to therapy. Curr Opin Neurol 31: 517-525, 2018.

23. Breiner A, Widdifield J, Katzberg HD, Barnett C, Bril V and Tu K: Epidemiology of myasthenia gravis in Ontario, Canada. Neuromuscul Disord 26: 41-46, 2016.

24. Kordas G, Lagoumintzis G, Sideris S, Poulas K and Tzartos SJ: Direct proof of the in vivo pathogenic role of the AChR autoantibodies from myasthenia gravis patients. PLoS One 9: e108327, 2014

25. Sala TP, Crave JC, Duracinsky M, Lepira Bompeka F, Tadmouri A, Chassany $\mathrm{O}$ and Cherin P: Efficacy and patient satisfaction in the use of subcutaneous immunoglobulin immunotherapy for the treatment of auto-immune neuromuscular diseases. Autoimmun Rev 17: 873-881, 2018.

26. Khedraki A, Reed EJ, Romer SH, Wang Q, Romine W, Rich MM, Talmadge RJ and Voss AA: Depressed synaptic transmission and reduced vesicle release sites in Huntington's disease neuromuscular junctions. J Neurosci 37: 8077-8091, 2017.

27. Verschuuren J, Strijbos E and Vincent A: Neuromuscular junction disorders. Handb Clin Neurol 133: 447-466, 2016.

28. Gonzalez-Freire M, de Cabo R, Studenski SA and Ferrucci L: The neuromuscular junction: Aging at the crossroad between nerves and muscle. Front Aging Neurosci 6: 208, 2014.

29. Zhang SJ, Li XX, Yu Y, Chiu AP, Lo LH, To JC, Rowlands DK and Keng VW: Schwann cell-specific PTEN and EGFR dysfunctions affect neuromuscular junction development by impairing agrin signaling and autophagy. Biochem Biophys Res Commun 515: $50-56,2019$.

30. Liu W, Klose A, Forman S, Paris ND, Wei-LaPierre L, Cortés-Lopéz M, Tan A, Flaherty M, Miura P, Dirksen RT and Chakkalakal JV: Loss of adult skeletal muscle stem cells drives age-related neuromuscular junction degeneration. Elife 6: e26464, 2017.

31. Pasnoor M, Dimachkie MM, Farmakidis C and Barohn RJ Diagnosis of myasthenia gravis. Neurol Clin 36: 261-274, 2018.

32. Özkök E, Durmuş H, Yetimler B, Taşlı H, Trakas N, Ulusoy C, Lagoumintzis G, Tzartos S and Tüzün E: Reduced muscle mitochondrial enzyme activity in MuSK-immunized mice. Clin Neuropathol 34: 359-363, 2015.

33. Chang DT and Reynolds IJ: Mitochondrial trafficking and morphology in healthy and injured neurons. Prog Neurobiol 80: 241-268, 2006
34. Sorrentino V,Menzies KJ and Auwerx J: Repairing mitochondrial dysfunction in disease. Annu Rev Pharmacol 58: 353-389, 2018.

35. Chaturvedi RK, Calingasan NY, Yang L, Hennessey T, Johri A and Beal MF: Impairment of PGC-1alpha expression, neuropathology and hepatic steatosis in a transgenic mouse model of Huntington's disease following chronic energy deprivation. Hum Mol Genet 19: 3190-3205, 2010.

36. Theilen NT, Kunkel GH and Tyagi SC: The role of exercise and TFAM in preventing skeletal muscle atrophy. J Cell Physiol 232: 2348-2358, 2017

37. Gorgey AS, Witt O, O'Brien L, Cardozo C, Chen Q, Lesnefsky EJ and Graham ZA: Mitochondrial health and muscle plasticity after spinal cord injury. Eur J Appl Physiol 119: 315-331, 2019.

38. Klinge CM: Estrogenic control of mitochondrial function and biogenesis. J Cell Biochem 105: 1342-1351, 2008.

39. Fukunaga K, Shinoda Y and Tagashira H: The role of SIGMAR1 gene mutation and mitochondrial dysfunction in amyotrophic lateral sclerosis. J Pharmacol Sci 127: 36-41, 2015.

40. Walczak J, Dębska-Vielhaber G, Vielhaber S, Szymański J, Charzyńska A, Duszyński J and Szczepanowska J: Distinction of sporadic and familial forms of ALS based on mitochondrial characteristics. FASEB J 33: 4388-4403, 2019.

41. Jésus P, Fayemendy P, Nicol M, Lautrette G, Sourisseau H, Preux PM, Desport JC, Marin B and Couratier P: Hypermetabolism is a deleterious prognostic factor in patients with amyotrophic lateral sclerosis. Eur J Neurol 25: 97-104, 2018.

42. Europa TA, Nel M and Heckmann JM: A review of the histopathological findings in myasthenia gravis: Clues to the pathogenesis of treatment-resistance in extraocular muscles. Neuromuscul Disord 29: 381-387, 2019.

43. Wu H, She S, Liu Y, Xiong W, Guo Y, Fang H, Chen H and Li J: Protective effect of Sijunzi decoction on neuromuscular junction ultrastructure in autoimmune myasthenia gravis rats. J Tradit Chin Med 33: 669-673, 2013

44. Vercauteren K, Gleyzer N and Scarpulla RC: PGC-1-related coactivator complexes with HCF-1 and NRF-2beta in mediating NRF-2(GABP)-dependent respiratory gene expression. J Biol Chem 283: 12102-12111, 2008.

45. Taherzadeh-Fard E, Saft C, Akkad DA, Wieczorek S, Haghikia A, Chan A, Epplen JT and Arning L: PGC-1alpha downstream transcription factors NRF-1 and TFAM are genetic modifiers of Huntington disease. Mol Neurodegener 6: 32, 2011.

46. Finsterer J, Oberman I and Reitner A: Respiratory chain complex-I defect mimicking myasthenia. Metab Brain Dis 17: 41-46, 2002.

47. Shichijo K, Mitsui T, Kunishige M, Kuroda Y, Masuda K and Matsumoto T: Involvement of mitochondria in myasthenia gravis complicated with dermatomyositis and rheumatoid arthritis: A case report. Acta Neuropathol 109: 539-542, 2005.

48. Kjøbsted R, Hingst JR, Fentz J, Foretz M, Sanz MN, Pehmøller C, Shum M, Marette A, Mounier R, Treebak JT, et al: AMPK in skeletal muscle function and metabolism. FASEB J 32: 1741-1777, 2018.

49. Zhang MH, Fang XS, Guo JY and Jin Z: Effects of AMPK on apoptosis and energy metabolism of gastric smooth muscle cells in rats with diabetic gastroparesis. Cell Biochem Biophys 77: 165-177, 2019.

50. Garcia-Carrizo F, Nozhenko Y, Palou A and Rodriguez AM: Leptin effect on acetylation and phosphorylation of Pgcla in muscle cells associated with Ampk and Akt activation in high-glucose medium. J Cell Physiol 231: 641-649, 2016.

51. Tamás P, Hawley SA, Clarke RG, Mustard KJ, Green K, Hardie DG and Cantrell DA: Regulation of the energy sensor AMP-activated protein kinase by antigen receptor and $\mathrm{Ca} 2+$ in $\mathrm{T}$ lymphocytes. J Exp Med 203: 1665-1670, 2006.

52. Martignago S, Fanin M, Albertini E, Pegoraro E and Angelini C: Muscle histopathology in myasthenia gravis with antibodies against MuSK and AChR. Neuropathol Appl Neurobiol 35: 103-110, 2009.

53. Willows R, Sanders MJ, Xiao B, Patel BR, Martin SR, Read J, Wilson JR, Hubbard J, Gamblin SJ and Carling D: Phosphorylation of AMPK by upstream kinases is required for activity in mammalian cells. Biochem J 474: 3059-3073, 2017.

54. Ke R, Xu Q, Li C, Luo L and Huang D: Mechanisms of AMPK in the maintenance of ATP balance during energy metabolism. Cell Biol Int 42: 384-392, 2018.

55. Inata Y, Kikuchi S, Samraj RS, Hake PW, O'Connor M, Ledford JR, O'Connor J, Lahni P, Wolfe V, Piraino G and Zingarelli B: Autophagy and mitochondrial biogenesis impairment contribute to age-dependent liver injury in experimental sepsis: dysregulation of AMP-activated protein kinase pathway. FASEB J 32: 728-741, 2018. 
56. Melser S, Lavie J and Benard G: Mitochondrial degradation and energy metabolism. Biochim Biophys Acta 1853: 2812-2821, 2015.

57. Cui Y, Chang L, Wang C, Han X, Mu L, Hao Y, Liu C, Zhao J, Zhang T, Zhang H, et al: Metformin attenuates autoimmune disease of the neuromotor system in animal models of myasthenia gravis. Int Immunopharmacol 75: 105822, 2019.

58. Nillni EA: The metabolic sensor Sirtl and the hypothalamus: Interplay between peptide hormones and pro-hormone convertases. Mol Cell Endocrinol 438: 77-88, 2016.

59. Xu YH, Song QQ, Li C, Hu YT, Song BB, Ye JM, Rao Y and Huang ZS: Bouchardatine suppresses rectal cancer in mice by disrupting its metabolic pathways via activating the SIRT1-PGC-1 $\alpha$-UCP2 axis. Eur J Pharmacol 854: 328-337, 2019.

60. Jang SY, Kang HT and Hwang ES: Nicotinamide-induced mitophagy: Event mediated by high $\mathrm{NAD}^{+} / \mathrm{NADH}$ ratio and SIRT1 protein activation. J Biol Chem 287: 19304-19314, 2012.

61. Snyder-Warwick AK, Satoh A, Santosa KB, Imai S and Jablonka-Shariff A: Hypothalamic Sirt1 protects terminal Schwann cells and neuromuscular junctions from age-related morphological changes. Aging Cell 17: e12776, 2018

62. Wu B, Feng JY, Yu LM, Wang YC, Chen YQ, Wei Y, Han JS, Feng X, Zhang Y, Di SY, et al: Icariin protects cardiomyocytes against ischaemia/reperfusion injury by attenuating sirtuin 1-dependent mitochondrial oxidative damage. Br J Pharmacol 175: 4137-4153, 2018.

63. Li Y, Xu S, Li J, Zheng L, Feng M, Wang X, Han K, Pi H, Li M, Huang $\mathrm{X}$, et al: SIRT1 facilitates hepatocellular carcinoma metastasis by promoting PGC- $1 \alpha$-mediated mitochondrial biogenesis. Oncotarget 7: 29255-29274, 2016.

64. Johnson ML, Robinson MM and Nair KS: Skeletal muscle aging and the mitochondrion. Trends Endocrinol Metab 24: 247-256, 2013.

65. Salt IP and Hardie DG: AMP-activated protein kinase an ubiquitous signaling pathway with key roles in the cardiovascular system. Circ Res 120: 1825-1841, 2017.

66. Akimoto T, Pohnert SC, Li P, Zhang M, Gumbs C, Rosenberg PB, Williams RS and Yan Z: Exercise stimulates Pgc-1alpha transcription in skeletal muscle through activation of the p38 MAPK pathway. J Biol Chem 280: 19587-19593, 2005.

67. Ljubicic V, Burt M and Jasmin BJ: The therapeutic potential of skeletal muscle plasticity in Duchenne muscular dystrophy: Phenotypic modifiers as pharmacologic targets. FASEB J 28 : 548-568, 2014

68. Abrahan $\mathrm{C}$ and Ash JD: The potential use of PGC-1 $\alpha$ and PGC-1 $\beta$ to protect the retina by stimulating mitochondrial repair. Adv Exp Med Biol 854: 403-409, 2016

69. Shu JT, Xu WJ, Zhang M, Song WT, Shan YJ, Song C, Zhu WQ, Zhang XY and Li HF: Transcriptional co-activator PGC-1 $\alpha$ gene is associated with chicken skeletal muscle fiber types. Genet Mol Res 13: 895-905, 2014.

70. Jiang SN, Teague AM, Tryggestad JB and Chernausek SD: Role of microRNA-130b in placental PGC-1 $\alpha /$ TFAM mitochondrial biogenesis pathway. Biochem Biophys Res Commun 487: 607-612, 2017

71. Felszeghy S, Viiri J, Paterno JJ, Hyttinen JMT, Koskela A, Chen M, Leinonen H, Tanila H, Kivinen N, Koistinen A, et al Loss of NRF-2 and PGC-1 $\alpha$ genes leads to retinal pigment epithelium damage resembling dry age-related macular degeneration. Redox Biol 20: 1-12, 2019.

72. Du H, Zhou C, Wu H, Shan T, Wu Z, Xu B and Zhang Y: Effects of electroacupuncture on PGC-1 $\alpha$ expression in brown adipose tissue. Evid Based Complement Alternat Med 2013: 625104 2013.

73. Cooper MP, Uldry M, Kajimura S, Arany Z and Spiegelman BM: Modulation of PGC-1 coactivator pathways in brown fat differentiation through LRP130. J Biol Chem 283: 31960-31967, 2008

74. Lehman JJ, Barger PM, Kovacs A, Saffitz JE, Medeiros DM and Kelly DP: Peroxisome proliferator-activated receptor gamma coactivator-1 promotes cardiac mitochondrial biogenesis 106 : $847-856,2000$

75. Zhang Q and Liang XC: Effects of mitochondrial dysfunction via AMPK/PGC-1 $\alpha$ signal pathway on pathogenic mechanism of diabetic peripheral neuropathy and the protective effects of Chinese medicine. Chin J Integr Med 25: 386-394, 2019.

76. Jones AW, Yao Z, Vicencio JM, Karkucinska-Wieckowska A and Szabadkai G: PGC-1 family coactivators and cell fate: Roles in cancer, neurodegeneration, cardiovascular disease and retrograde mitochondria-nucleus signalling. Mitochondrion 12: $86-99,2012$
77. Xiang Z, Valenza M, Cui L, Leoni V, Jeong HK, Brilli E, Zhang J, Peng Q, Duan W, Reeves SA, et al: Peroxisome-proliferator-activated receptor gamma coactivator $1 \alpha$ contributes to dysmyelination in experimental models of Huntington's disease. J Neurosci 31: 9544-9553, 2011.

78. Wang Y, Zhao X, Lotz M, Terkeltaub R and Liu-Bryan R: Mitochondrial biogenesis is impaired in osteoarthritis chondrocytes but reversible via peroxisome proliferator-activated receptor $\gamma$ coactivator $1 \alpha$. Arthritis Rheumatol 67: 2141-2153, 2015.

79. Koh JH, Hancock CR, Terada S, Higashida K, Holloszy JO and Han DH: PPAR $\beta$ is essential for maintaining normal levels of PGC-1 $\alpha$ and mitochondria and for the increase in muscle mitochondria induced by exercise. Cell Metab 25: 1176-1185 e5, 2017.

80. Hsieh PF, Liu SF, Hung TJ, Hung CY, Liu GZ, Chuang LY, Chen MF, Wang JL, Shi MD, Hsu CH, et al: Elucidation of the therapeutic role of mitochondrial biogenesis transducers NRF-1 in the regulation of renal fibrosis. Exp Cell Res 349: 23-31, 2016.

81. Lanza IR and Nair KS: Regulation of skeletal muscle mitochondrial function: Genes to proteins. Acta Physiol (Oxf) 199: 529-547, 2010.

82. Ramachandran B, Yu GS and Gulick T: Nuclear respiratory factor 1 controls myocyte enhancer factor $2 \mathrm{~A}$ transcription to provide a mechanism for coordinate expression of respiratory chain subunits. J Biol Chem 283: 11935-11946, 2008.

83. Matsuda T, Kanki T, Tanimura T, Kang D and Matsuura ET: Effects of overexpression of mitochondrial transcription factor A on lifespan and oxidative stress response in Drosophila melanogaster. Biochem Biophys Res Commun 430: 717-721, 2013.

84. Thirupathi A and Pinho RA: Effects of reactive oxygen species and interplay of antioxidants during physical exercise in skeletal muscles. J Physiol Biochem 74: 359-367, 2018.

85. Brandt N, Dethlefsen MM, Bangsbo J and Pilegaard H: PGC-1a and exercise intensity dependent adaptations in mouse skeletal muscle. PLoS One 12: e0185993, 2017.

86. Wu KLH, Wu CW, Chao YM, Hung CY and Chan JYH: Impaired Nrf2 regulation of mitochondrial biogenesis in rostral ventrolateral medulla on hypertension induced by systemic inflammation. Free Radic Biol Med 97: 58-74, 2016.

87. Hu Q, Ren J, Li G, Wu J, Wu X, Wang G, Gu G, Ren H, Hong Z and Li J: The mitochondrially targeted antioxidant MitoQ protects the intestinal barrier by ameliorating mitochondrial DNA damage via the Nrf2/ARE signaling pathway. Cell Death Dis 9: 403, 2018.

88. Bernard K, Logsdon NJ, Miguel V, Benavides GA, Zhang J, Carter AB, Darley-Usmar VM and Thannickal VJ: NADPH oxidase 4 (Nox4) suppresses mitochondrial biogenesis and bioenergetics in lung fibroblasts via a nuclear factor erythroid-derived 2-like 2 (Nrf2)-dependent pathway. J Biol Chem 292: 3029-3038, 2017.

89. Kang I, Chu CT and Kaufman BA: The mitochondrial transcription factor TFAM in neurodegeneration: Emerging evidence and mechanisms. FEBS Lett 592: 793-811, 2018.

90. Piao Y, Kim HG, Oh MS and Pak YK: Overexpression of TFAM, NRF-1 and myr-AKT protects the MPP $(+)$-induced mitochondrial dysfunctions in neuronal cells. Biochim Biophys Acta 1820: 577-585, 2012

91. Rostedt Punga A, Ahlqvist K, Bartoccioni E, Scuderi F, Marino M, Suomalainen A, Kalimo H and Stålberg EV: Neurophysiological and mitochondrial abnormalities in MuSK antibody seropositive myasthenia gravis compared to other immunological subtypes. Clin Neurophysiol 117: 1434-1443, 2006.

92. Kunkel GH, Chaturvedi P and Tyagi SC: Mitochondrial pathways to cardiac recovery: TFAM. Heart Fail Rev 21: 499-517, 2016.

93. Ruzzenente B, Rötig A and Metodiev MD: Mouse models for mitochondrial diseases. Hum Mol Genet 25: R115-R122, 2016.

94. Li H, Slone J, Fei L and Huang T: Mitochondrial DNA variants and common diseases: A mathematical model for the diversity of age-related mtDNA mutations. Cells 8: 608, 2019.

95. Lezza AMS: Mitochondrial transcription factor A (TFAM): One actor for different roles. Front Biol 7: 30-39, 2012.

96. Xu S, Zhong M, Zhang L, Wang Y, Zhou Z, Hao Y, Zhang W, Yang X, Wei A, Pei L and Yu Z: Overexpression of Tfam protects mitochondria against beta-amyloid-induced oxidative damage in SH-SY5Y cells. FEBS J 276: 3800-3809, 2009.

97. Kang D, Kim SH and Hamasaki N: Mitochondrial transcription factor A (TFAM): Roles in maintenance of mtDNA and cellular functions. Mitochondrion 7: 39-44, 2007. 
98.Dong J, Zhao J, Zhang M, Liu G, Wang X, Liu Y, Yang N, Liu Y, Zhao G, Sun J, et al: $\beta 3$-Adrenoceptor impairs mitochondrial biogenesis and energy metabolism during rapid atrial pacing-induced atrial fibrillation. J Cardiovasc Pharmacol Ther 21: 114-126, 2016.

99. Tao L, Wang L, Yang X, Jiang X and Hua F: Recombinant human glucagon-like peptide-1 protects against chronic intermittent hypoxia by improving myocardial energy metabolism and mitochondrial biogenesis. Mol Cell Endocrinol 481: 95-103, 2019.

100. Jeon SM: Regulation and function of AMPK in physiology and diseases. Exp Mol Med 48: e245, 2016.

101. Aguirre-Rueda D, Guerra-Ojeda S, Aldasoro M, Iradi A, Obrador E, Ortega A, Mauricio MD, Vila JM and Valles SL: Astrocytes protect neurons from A $\beta 1-42$ peptide-induced neurotoxicity increasing TFAM and PGC-1 and decreasing PPAR- $\gamma$ and SIRT-1. Int J Med Sci 12: 48-56, 2015.

102. Hood DA, Tryon LD, Carter HN, Kim Y and Chen CCW: Unravelling the mechanisms regulating muscle mitochondrial biogenesis. Biochem J 473: 2295-2314, 2016.

103. Nie Y, Sato Y, Wang C, Yue F, Kuang S and Gavin TP: Impaired exercise tolerance, mitochondrial biogenesis, and muscle fiber maintenance in miR-133a-deficient mice. FASEB J 30 3745-3758, 2016.

104. Nirwane A and Majumdar A: Understanding mitochondrial biogenesis through energy sensing pathways and its translation in cardio-metabolic health. Arch Physiol Biochem 124: 194-206, 2018.

105.Dhar SS and Wong-Riley MTT: Coupling of energy metabolism and synaptic transmission at the transcriptional level: Role of nuclear respiratory factor 1 in regulating both Cytochrome c oxidase and NMDA glutamate receptor subunit genes. J Neurosci 29: 483-492, 2009.

106. Huang DD, Fan SD, Chen XY, Yan XL, Zhang XZ, Ma BW, Yu DY, Xiao WY, Zhuang CL and Yu Z: Nrf2 deficiency exacerbates frailty and sarcopenia by impairing skeletal muscle mitochondrial biogenesis and dynamics in an age-dependent manner. Exp Gerontol 119: 61-73, 2019.

107. Chuang YC, Chen SD, Jou SB, Lin TK, Chen SF, Chen NC and Hsu CY: Sirtuin 1 regulates mitochondrial biogenesis and provides an endogenous neuroprotective mechanism against seizure-induced neuronal cell death in the hippocampus following status epilepticus. Int J Mol Sci 20: 3588, 2019.

108. Van Laar VS, Arnold B, Howlett EH, Calderon MJ, St Croix CM, Greenamyre JT, Sanders LH and Berman SB Evidence for compartmentalized axonal mitochondrial biogenesis: Mitochondrial DNA replication increases in distal axons as an early response to Parkinson's disease-relevant stress. J Neurosci 38: 7505-7515, 2018.

109. Osborne B, Cooney GJ and Turner N: Are sirtuin deacylase enzymes important modulators of mitochondrial energy metabolism? Biochim Biophys Acta 1840: 1295-1302, 2014.

110. Jarmuszkiewicz W and Szewczyk A: Energy-dissipating hub in muscle mitochondria: Potassium channels and uncoupling proteins. Arch Biochem Biophys 664: 102-109, 2019.

111. Dan Dunn J, Alvarez LA, Zhang X and Soldati T: Reactive oxygen species and mitochondria: A nexus of cellular homeostasis. Redox Biol 6: 472-485, 2015.

112. Rahman S and Hanna MG: Diagnosis and therapy in neuromuscular disorders: Diagnosis and new treatments in mitochondrial diseases. J Neurol Neurosurg Psychiatry 80: 943-953, 2009.

113. Cabezas R, Baez-Jurado E, Hidalgo-Lanussa O, Echeverria V, Ashrad GM, Sahebkar A and Barreto GE: Growth factors and neuroglobin in astrocyte protection against neurodegeneration and oxidative stress. Mol Neurobiol 56: 2339-2351, 2019.

114. Niemann A, Huber N, Wagner KM, Somandin C, Horn M Lebrun-Julien F, Angst B, Pereira JA, Halfter H, Welzl H, et al: The Gdap1 knockout mouse mechanistically links redox control to charcot-marie-tooth disease. Brain 137: 668-682, 2014.

115.Li Y, Zhao X, Hu Y, Sun H, He Z, Yuan J, Cai H, Sun Y, Huang X, Kong W and Kong W: Age-associated decline in Nrf2 signaling and associated mtDNA damage may be involved in the degeneration of the auditory cortex: Implications for central presbycusis. Int J Mol Med 42: 3371-3385, 2018.
116. Li X, Fang P, Yang WY, Chan K, Lavallee M, Xu K, Gao T, Wang $\mathrm{H}$ and Yang X: Mitochondrial ROS, uncoupled from ATP synthesis, determine endothelial activation for both physiological recruitment of patrolling cells and pathological recruitment of inflammatory cells. Can J Physiol Pharmacol 95: 247-252, 2017.

117. Danikowski KM, Jayaraman S and Prabhakar BS: Regulatory $\mathrm{T}$ cells in multiple sclerosis and myasthenia gravis J Neuroinflammation 14: 117, 2017

118. Tarnopolsky M, Brady L and MacNeil L: Myasthenia graves-like symptoms associated with rare mitochondrial mutation (m.5728T >C). Mitochondrion 47:139-140, 2019.

119. Wang X and Rich MM: Homeostatic synaptic plasticity at the neuromuscular junction in myasthenia gravis. Ann NY Acad Sci 1412: 170-177, 2018

120. Lanser AJ, Rezende RM, Rubino S, Lorello PJ, Donnelly DJ, $\mathrm{Xu}$ H, Lau LA, Dulla CG, Caldarone BJ, Robson SC and Weiner HL: Disruption of the ATP/adenosine balance in CD39 ${ }^{-/}$mice is associated with handling-induced seizures. Immunology 152: 589-601, 2017.

121.Zhang Y and Xu H: Translational regulation of mitochondrial biogenesis. Biochem Soc Trans 44: 1717-1724, 2016.

122. Doan KN, Ellenrieder L and Becker T: Mitochondrial porin links protein biogenesis to metabolism. Curr Genet 65: 899-903, 2019.

123.Jeffery CJ: Enzymes, pseudoenzymes, and moonlighting proteins: Diversity of function in protein superfamilies. FEBS J, Jun 13, 2020 (Online ahead of print).

124. Askanas V, Engel WK and Nogalska A: Pathogenic considerations in sporadic inclusion-body myositis, a degenerative muscle disease associated with aging and abnormalities of myoproteostasis. J Neuropathol Exp Neurol 71: 680-693, 2012.

125. Arnold W, McGovern VL, Sanchez B, Li J, Corlett KM, Kolb SJ, Rutkove SB and Burghes AH: The neuromuscular impact of symptomatic SMN restoration in a mouse model of spinal muscular atrophy. Neurobiol Dis 87: 116-123, 2016.

126. Saifetiarova J, Liu X, Taylor AM, Li J and Bhat MA: Axonal domain disorganization in Caspr1 and Caspr2 mutant myelinated axons affects neuromuscular junction integrity, leading to muscle atrophy. J Neurosci Res 95: 1373-1390, 2017.

127. Wu H, She S, Liu Y, Xiong W, Guo Y, Fang H, Chen H and Li J: Protective effect of Sijunzi decoction on neuromuscular junction ultrastructure in autoimmune myasthenia gravis rats. J Tradit Chin Med 33: 669-673, 2013.

128. Attia M, Maurer M, Robinet M,Le Grand F, Fadel E, Le Panse R, Butler-Browne $\mathrm{G}$ and Berrih-Aknin S: Muscle satellite cells are functionally impaired in myasthenia gravis: Consequences on muscle regeneration. Acta Neuropathol 134: 869-888, 2017.

129. Iwasa K, Furukawa Y, Yoshikawa H and Yamada M: Caveolin-3 is aberrantly expressed in skeletal muscle cells in myasthenia gravis. J Neuroimmunol 301: 30-34, 2016.

130. Rivner MH, Pasnoor M, Dimachkie MM, Barohn RJ and Mei L: Muscle-specific tyrosine kinase and myasthenia gravis owing to other antibodies. Neurol Clin 36: 293-310, 2018.

131. Beecher G, Putko BN, Wagner AN and Siddiqi ZA: Therapies directed against $b$-cells and downstream effectors in generalized autoimmune myasthenia gravis: Current status. Drugs 79 353-364, 2019.

132. Valbuena GN, Rizzardini M, Cimini S, Siskos AP, Bendotti C, Cantoni L and Keun HC: Metabolomic analysis reveals increased aerobic glycolysis and amino acid deficit in a cellular model of amyotrophic lateral sclerosis. Mol Neurobiol 53: 2222-2240, 2016

133. Lysenko EA, Popov DV, Vepkhvadze TF, Lednev EM and Vinogradova OL: Effect of combined aerobic and strength exercise on regulation of mitochondrial biogenesis, protein synthesis and degradation in human skeletal muscle. Fiziol Cheloveka 42: 58-69, 2016 (In Russian).

This work is licensed under a Creative Commons Attribution-NonCommercial-NoDerivatives 4.0 International (CC BY-NC-ND 4.0) License. 